\title{
Anastomosis hepáticoduodenal. ¿Técnica de elección para el tratamiento de quiste de colédoco? Seguimiento a largo plazo de un estudio interinstitucional
}

\author{
Dr. Antonio Francisco Gallardo-Meza, ${ }^{1}$ Dr. José Manuel González-Sánchez, ${ }^{2}$ Dr. Miguel Ángel Piña-Garay, ${ }^{2}$ \\ Dr. Miguel Ángel Medina-Andrade, ${ }^{2}$ Dr. Raúl Villarroel-Cruz, ${ }^{2}$ Dr. Armando Martínez-de la Barquera, ${ }^{3}$ * \\ Dr. Leopoldo Torres-Contreras ${ }^{4 *}$
}

\section{RESUMEN}

El tratamiento de elección del quiste de colédoco es su resección con una técnica de reconstrucción bilioentérica, ya sea la anastomosis hepáticoduodenal o la anastomosis hepáticoyeyunal en Y de Roux. La primera cayó en desuso porque se complicaba frecuentemente de colangitis ascendente.

Estudiamos 15 casos, operados en tres instituciones del país con un seguimiento promedio de ocho años; ningún caso se complicó de colangitis ascendente; por esta razón consideramos que esta técnica se debe volver a reconsiderar como una alternativa útil y confiable para el tratamiento de quiste de colédoco.

Palabras clave: Quiste de colédoco, anastomosis hepáticoduodenal, anastomosis en Y de Roux, reconstrucción bilioentérica.

\section{ABSTRACT}

The treatment of choice of choledochal cyst is the resection of the cyst and a enterobiliary reconstruction. Most authors prefer the hepaticjejuno anastomosis with a $Y$ Roux operation instead of hepatic-duodenal anastomosis because it is seldom complicated with ascendant cholangitis. We present our result in 15 patients operated with a hepatic-duodenal anastomosis, which were operated in three different institutions in our country, with a follow-up of 8 years and without documented cases of ascendant cholangitis. We believe this should be re-considered as a useful technique for the treatment of choledochal cyst.

Key words: Choledochal cyst, hepatic-duodenal anastomosis, Y Roux operation, enterobiliary reconstruction.



1 quiste de colédoco es una dilatación congénita del árbol biliar intra o extrahepático. Esta afección es infrecuente. En los países occidentales la frecuencia del quiste de colédoco es de un caso por cada 100,000 a 150,000 nacidos vivos, de los cuales $75 \%$ se diagnostica en la niñez y $20 \%$ en el adulto. La relación hombre mujer varía entre $3: 1$ y $4: 1^{1-5}$. El trata-

Jefe de Cirugía Pediátrica. Hospital General de Occidente, Guadalajara, Jal.

2 Adscrito a Cirugía Pediátrica. Hospital General de Occidente. Guadalajara, Jal.

3 Adscrito a Cirugía Pediátrica. Hospital Vicente Guerrero, Acapulco, Gro.

4 Jefe de Cirugía Pediátrica. Hospital Central Sur Picacho PEMEX. México D.F.

* Agradecemos a los Drs. Martínez-de la Barquera y TorresContreras, su contribución con casos atendidos en sus respectivas instituciones. miento de elección del quiste de colédoco es su resección y la reconstrucción bilioentérica, ya sea la anastomosis hepáticoduodenal o la anastomosis hepáticoyeyunal en $\mathrm{Y}$ de Roux ${ }^{6-12}$. La primera cayó en desuso por haber propiciado colangitis ascendente en muchos casos; sin embargo, algunos autores como Todani a partir de 1979 señala que la frecuencia de colangitis es similar a la que se ve en la

Correspondencia: Dr. Antonio Francisco Gallardo-Meza. Hospital General de Occidente. Av. Zoquipan 1050. Col. Seattle. Zapopan, Jalisco. C.P. 45170. Tel y Fax: (33) 30306354.

E-mail: antoniogallardo_@hotmail.com

Recibido: julio, 2009. Aceptado: noviembre, 2009.

Este artículo debe citarse: Gallardo-Meza AF, González-Sánchez JM, Piña-Garay MA y col. Anastomosis hepáticoduodenal. ¿Técnica de elección para el tratamiento de quiste de colédoco? Seguimiento a largo plazo de un estudio interinstitucional. Acta Pediatr Mex 2010;31(1):3-5.

La versión completa de este artículo también está disponible en: www.nietoeditores.com.mx 
anastomosis hepáticoyeyunal en Y de Roux ${ }^{10-12}$. En el año 2001 Gallardo Meza, Villarroel Cruz, y cols. ${ }^{13}$, publicamos seis casos de quiste del colédoco tratados con resección del quiste y anastomosis hepáticoduodenal como técnica de reconstrucción bilioentérica, con un seguimiento a tres años sin colangitis ascendente. En el Congreso Nacional de Cirugía Pediátrica de Cancún, Quintana Roo, en el 2003 presentamos tres casos más, haciendo un total de nueve pacientes operados con esta técnica y con seguimiento promedio de cinco años sin colangitis, incluyendo un caso operado en el Hospital Vicente Guerrero de Acapulco. Actualmente presentamos 15 casos en tres instituciones diferentes de la República Mexicana, con seguimiento promedio de ocho años y en ningún caso se ha documentado colangitis ascendente.

\section{MATERIAL Y METODO}

Se evaluaron 15 pacientes de tres instituciones de la República Mexicana, 11 del Hospital General de Occidente de Guadalajara, Jal., dos del hospital Vicente Guerrero en Acapulco, Gro.; dos del Hospital Central Sur Picacho de PEMEX. Fueron diez mujeres y cinco hombres. Su edad iba de tres meses a 14 años. El método diagnóstico fue con ultrasonido abdominal (US) en los 15 pacientes; se realizó pancreato-colangiografía transduodenoscópica en cuatro casos que tenían pancreatitis grave ${ }^{21}$. En dos más se llevó a cabo una colangio resonancia y a diez se les realizó TAC abdominal. A todos se les hizo resección total del quiste, dejando un muñón del hepático común a la altura de la bifurcación del hepático derecho e izquierdo, sitio en el cual se realizó la anastomosis hepáticoduodenal termino lateral en un solo plano con puntos separados de material absorbible de Vicryl 5-0; se dejó una boca anastomótica de cuando menos $2 \mathrm{~cm}$ de diámetro. A 12 pacientes se les dejo una sonda en $\mathrm{T}$ en el sitio de la anastomosis para valorar reflujo duodeno biliar. El inicio de la vía oral fue a los cinco días en todos los casos; a los que se les dejo una sonda en $\mathrm{T}$ se les realizó una colangiografía trans-sonda a los 21 días para corroborar la permeabilidad de la anastomosis y la ausencia de fuga biliar, tiempo en el cual se retiró la sonda. A todos los pacientes se les hizo seguimiento con US abdominal y pruebas de función hepática a los tres y seis meses del postoperatorio y posteriormente cada seis meses en los tres primeros años, después de este tiempo, cada año.

\section{RESULTADOS}

El US es el estudio de elección para hacer el diagnóstico; en algunos casos se complementa con TAC o con colangioresonancia ${ }^{14}$. No encontramos ventaja en hacer la conlangiopancreatografía transduodenoscópica, ya que este estudio requiere anestesia general y no aportó más datos que los de una TAC o una colangioresonancia. En nuestros casos predominó el sexo femenino: 2:1. La edad al momento del diagnóstico que fue el mismo tiempo de la cirugía, varió de tres meses a 14 años, con una media de cinco años. La evolución postoperatoria fue excelente en los 15 casos. En los pacientes en quienes se dejó una sonda en T (12 casos), no ocurrió reflujo biliar a través de la misma. El seguimiento a largo plazo fue de seis meses, en el caso más recientemente operado hasta 16 años, con un promedio de ocho años. No se registraron episodios de colangitis en ningún paciente por clínica ni por laboratorio. Las pruebas de función hepática fueron normales. En 11 casos se encontró aire en la vía biliar, lo que no tuvo traducción clínica ni de laboratorio.

\section{DISCUSIÓN}

La dilatación sacular de un quiste del colédoco causa acumulación de bilis, lo que propicia la formación de litiasis, estenosis; esto a su vez ocasiona el desarrollo de pancreatitis o colangitis graves. Hay muchas teorías sobre la etiología de los quistes biliares ya que no han sido probadas ${ }^{15-20}$. Existe gran controversia sobre el origen de los quistes, sobre si son congénitos o adquiridos.

Estudios recientes tratan de explicar los defectos quísticos de la vía biliar por una reducción de la cantidad normal de los cuerpos de células postcolinérgicas, es decir, oligoganglionosis en la porción distal del colédoco. Lo comprobaron contando los cuerpos de las células ganglionares en pacientes con quistes del colédoco y en pacientes con colédocos normales; encontraron una disminución significativa del número de células en la porción distal de colédocos dilatados ${ }^{16-20}$.

A diferencia de lo que se ha publicado, en nuestra experiencia la anastomosis hepáticoduodenal es una opción de primera elección, ya que no hemos observado datos clínicos, como fiebre e ictericia. Todani et al. han utilizado la anastomosis hepáticoduodenal con buenos resultados, aun cuando es la misma frecuencia de colangitis que en 
la anastomosis hepáticoyeyunal ${ }^{11-12}$. El hallazgo de aire en la vía biliar en los estudios de US de control, nos hace pensar que probablemente sí exista paso de material duodenal hacia la vía biliar intrahepática, pero si la anastomosis hepáticoduodenal es amplia como en nuestros casos, el aclaramiento del contenido duodeno biliar debe ser inmediato, por lo que no hay acumulación del mismo en la vía biliar residual (hepático común, hepático derecho o izquierdo), con lo que se evita la colangitis ${ }^{14}$; esto queda demostrado con la falta de datos clínicos y de laboratorio.

\section{CONCLUSIONES}

Pensamos que la anastomosis hepáticoduodenal como técnica de reconstrucción biliar en pacientes con quiste de colédoco es una alternativa confiable y de primera elección. Es técnicamente más fácil de realizar que la anastomosis hepáticoyeyunal en Y de Roux, ya que prácticamente no hay movilización de asas y por ende menos adherencias postoperatorias ni colangitis ascendente en todos los casos estudiados.

\section{REFERENCIAS}

1. Todani T, Wantanabe $\mathrm{Y}$, Narsue M, et al. Congenital bile duch cysts, classification, operative procedures and review of thirtyseven cases, including cancer araising from choledochal cyst. Am J Surg. 1977;134:263-9.

2. Alonso-Lej F, Rever WB, Pessangno DJ. Congenital choledococele cyst, with a report of 2 analysis of 94 cases. Surg Gynecol Obstet. 1959;108:1-30.

3. Shi LB, Peng SY, Meg XK, Peng CH, Lui YB, Chen XP, et al. Diagnosis and treatment of congenital choledochal cyst: 20 year experience in China. World J Gastroenterol. 2001;7(5):732-4.

4. Robertson J, Raine P. Choledocahl cyst: A 33-year review. $\mathrm{Br}$ J Surg. 1988;75:799-801.

5. Flanigan P. Biliary cyst. Ann Surg. 1975;5:635-49.

6. Scudamore $\mathrm{CH}$, Hemming AW, Teare JP, Fache JS, ErbSR, Watkinson AF. Surgical management of choledochal cyst. Am J Surg. 1994;167:497-500.
7. Chijiwa K, Koga A. Surgical management and long-term follow up of patients with choledochal cyst. Am J Surg. 1993;165:23842.

8. Barlow B, Tabor E, Blanc WA, Santulli TV, Harris RC. Choledochal cyst: a review of 19 Cases. J Pediatr. 1976;89:940-8.

9. Filler R, Stringer $\mathrm{G}$. Treatment of choledochal cyst by excision. J Pediatr Surg. 1980;15:437-42

10. Todani T, Watanabe $\mathrm{Y}$, Fuji T, et al. Congenital choledochal cyst with intrahepatic involvement. Arch Surg. 1984;119:103843.

11. Todani T, Watanabe Y, Mizuguchi T, Fuji T, Toki A. Hepaticoduodenostomy at the hepatic hilum after excision of choledochal cyst. Am J Surg. 1981;142:584-87.

12. Shimotakahara A, Yamataka A, Yani T, et al. Roux-in-Y hepaticojejunostomy or hepaticoduodenostomy for biliary reconstruction during surgical treatment of choledochal cyst: which is better? Pediatr Surg Int. 2005;21:5-7.

13. Gallardo Meza AF, Villaroel Cruz R, Piña Garay MA, Dessavre Dávila A, Palacios Zulueta Ma del C, Martínez González MT, Parra Muro M, Muñiz Sandoval I. Anastomosis hepaticoduodenal: una alternativa confiable en el tratamiento del quiste de colédoco. Rev Mex Cir Pediatr. 2001;8:84-87.

14. Ystgaard B, Myrvold HE, Pilsen G. Magnetic resonance imaging in preoperative assessment of choledochal cyst. Eur $\mathrm{J}$ Surg. 1992;158:567-9.

15. Yamataka A, Oshiro K, Okada Y, et al. Complications alter cyst excision with hepaticoenterostomy for choledochal cyst and their surgical management in children versus adults. J Pediatr Surg. 1997;32:1097-102.

16. Iwai N, Yanagihasa S, Tokiwa K, Shimatoke T, Nakamura K. Congenital choledochal dilatation with emphasis on pathophisiology of the biliary tract. Ann Surg. 1992;215(1):27-30.

17. O'Neill JA. Choledochal cyst. In: O’Neill JA, Rowe MI, Grosfeld $\mathrm{JL}$, Fonkalsrud EW, Coran AG. Pediatric Surgery. $5^{\text {th }}$ Edition. Missouri St. Louis: Mosby; 1998. p. 1483-94.

18. Sigalet DL. Trastornos de vías biliares e hipertensión portal. En: Ashcraft KL, Murphy JP, Sharp RJ, Sigalet DL, Zinder CL. Cirugía pediátrica. $3^{a}$ Edición. Philadelphia: WB Saunders; 2002. p. $610-28$.

19. Altman RP, Hicks BA. Choledochal cyst. In: Spitz L, Coran AG. $5^{\text {th }}$ Edition Pediatric Surgery. London: Chapman and Hall Medical; 1994. p. 562-69.

20. Karrer FM, Pence JC. Billiary atresia and choledochal cyst. In: Ziegler MM, Azizkhan RG, Weber TR. Operative Pediatric Surgery. Boston: Mc Graw-Hill; 2003. p. 775-88.

21. Garrido Torrecillas FJ, Avila Villegas R, Del Moral Romero E, Moreno Sánchez A. Quiste de colédoco asociado a pancreatitis aguda. Diagnóstico preoperatorio. Servicio de Pediatría Hospital Clínico Universitario San Cecilio Granada. An Pediatr. 2002;57:77-8. 